\title{
Non-Periodic and not Everywhere Dense Billiard Trajectories in Convex Polygons and Polyhedrons
}

\author{
G. A. Galperin \\ Department of Mathematics, Moscow State University, Moscow, USSR
}

\begin{abstract}
This paper proves the existence of non-periodic and not everywhere dense billiard trajectories in convex polygons and polyhedrons. For any $n \geqq 3$ there exists a corresponding convex $n$-agon (for $n=3$ this will be a right triangle with a small acute angle), while in three-dimensional space it will be a prism, the $n$-agon serving as the base.

The results are applied for investigating a mechanical system of two absolutely elastic balls on a segment, and also for proving the existence of an infinite number of periodic trajectories in the given polygons.

The exchange transformation of two intervals is used for proving the theorems. An arbitrary exchange transformation of any number of intervals can also be modeled by a billiard trajectory in some convex polygon with many sides.
\end{abstract}

\section{Introduction. Formulation of Theorems}

Billiards in a polygon $Q$ on the Euclidean plane $\mathbb{R}^{2}$ are a dynamical system. This system is defined by uniform motion of a point (particle) inside $Q$ with elastic reflections at the polygon's boundary $\partial Q$, such that the tangential component of the velocity remains constant and the normal component changes sign. We will assume that the magnitude of the particle's velocity equals one.

The motion described is not limited in time if the particle does not fall into a vertex of $Q$. Otherwise, its motion is determined only till it falls into a vertex; this is a special case and we will not consider it.

The phase space $\mathscr{M}=\mathscr{M}(Q)$ of this dynamical system is a subset of the direct product $Q \times S^{1}$ ( $S^{1}$ being the circle of unit velocities), which is obtained by identifying pairs $(q, v)$ and $\left(q, v^{\prime}\right)$ for $q \in \partial Q, v, v^{\prime} \in S^{1}$ and

$$
v-v^{\prime}=2(n, v) n,
$$

where $n=n(q)$ is the external unit vector normal to $\partial Q$ at point $q$.

A billiard system in a polyhedron is defined in the same way. 
Sometimes not only the billiard trajectory in the phase space $\mathscr{M}(Q)$ is called the trajectory in $Q$, but also its projection onto the configuration space $Q$, i.e., the trace of the moving particle in $Q$. As a rule, we will also call this projection in the configuration space a billiard trajectory, unless stated otherwise.

In this paper we will discuss individual billiard trajectories in polygons and polyhedrons, and in particular, in "rational" polygons in which all the angles are commensurate with $\pi$. In papers $[1,2]$ it is proved that for billiards in a rational polygon $Q$ almost every trajectory is either (a) periodic or (b) everywhere dense in $Q$ (for the remaining polygons this assertion is not proved). However, an arbitrarily chosen billiard trajectory in a polygon (not necessarily a rational one) can a priori be (c) non-periodic and not everywhere dense in $Q$. Such a trajectory must be everywhere dense in a subset of $Q$.

A natural question arises: Can all cases (a)-(c) be realized? For cases (a) and (b) a positive answer is obvious. For case (c), however, the answer is not yet clear. For a long time there was a hypothesis that any individual trajectory in any convex polygon suits either case (a) or case (b), i.e., the set of trajectories of case (c) is empty (according to an oral communication by Zemlyakov, author of paper [1]; also see his popular article [3]). This hypothesis was stated with a view to the following considerations.

From the point of view of analytical mechanics the billiard considered is a Hamiltonian system with two degrees of freedom. In the case of a rational polygon, this system has two independent first integrals, its phase space is stratified into invariant two-dimensional manifolds of some genus $g$ (pretzels), and studying the billiard in the polygon $Q$ is reduced to studying the windings of these manifolds [1]. As is known, for a torus $(g=1)$ such a winding is always either closed (then the trajectory in $Q$ is periodic) or everywhere dense on the torus (then the billiard trajectory is dense in $Q$ ), and thus there is no case (c). For example, billiards in the following simplest polygons are reduced to windings on a torus: a rectangle, an equilateral triangle, and right triangles with angles $\pi / 4$ and $\pi / 6$. Already in a triangle with angles $\pi / 2, \pi / 8,3 \pi / 8$, studying a billiard is not reduced to windings on a torus; it must be examined in a more complicated way. However, the statement of the hypothesis holds for this case too.

The well known example of a dynamical system consisting of two point particles with masses $m_{1}$ and $m_{2}$ moving freely on the interval $[0,1]$ and rebounding elastically from each other and from the endpoints of the interval (in accordance with the classical laws of an elastic collision; see [4]) leads us to consider a billiard in a right triangle. The configuration space of this system is a right triangle with acute angle $\alpha=\arctan \sqrt{\frac{m_{1}}{m_{2}}}$ and the system itself is equivalent to a billiard in this triangle. The collision of the point particles is represented by a configuration point reflecting from the hypotenuse, and the reflection of the particle from an endpoint is represented by the point's reflection from a cathetus. If $k=\frac{m_{1}}{m_{2}}$ has one of the following values: $1 ; 3 ;(3+2 \sqrt{2})^{2} ; 1 / 3 ;(3+2 \sqrt{2})^{-2}($ the $k$ 's correspond to angles $\alpha=\frac{\pi}{4} ; \frac{\pi}{3} ; \frac{\pi}{8} ; \frac{\pi}{6}$, and $\frac{3 \pi}{8}$ in the right triangle), then the 
configuration trajectory of the mechanical system described will be as in cases (a) and (b); here case (c) is absent.

In this paper the hypothesis is solved negatively. We prove that the set (c) of billiard trajectories, neither periodic nor everywhere dense in $Q$, is not empty.

We cite below the exact formulations of all the results obtained.

Theorem 1. For any $n \geqq 3$ there exists on the Euclidean plane $\mathbb{R}^{2}$ a convex $n$-agon $Q$ in which there is a non-periodic and not everywhere dense billiard trajectory $\Gamma$.

Theorem 2. For almost all $\alpha$ from some interval $\left(0, \alpha_{0}\right)$, there exists on the Euclidean plane $\mathbb{R}^{2}$ a right triangle with acute angle $\alpha$ in which there is a non-periodic and not everywhere dense billiard trajectory.

Theorem 3. Consider the dynamical system of two point particles elastically colliding on the interval $[0,1]$, with masses $m$ and $\mathrm{km}$. For almost all $k$ in some interval $\left(0, k_{0}\right)$, and with some initial state of the particles' positions and velocities, the set of points where they collide with each other fills everywhere densely the union of a finite number of non-intersecting intervals on $[0,1]$.

Theorem 4. In Euclidean three-dimensional space $\mathbb{R}^{3}$ there is a convex polyhedron containing a non-periodic billiard trajectory, which is not everywhere dense, but which is everywhere dense in a three dimensional region in this polyhedron.

The main idea of the proof of Theorems 1-3 consists of constructing a family of a parallel segments in the polygon, such that the billiard dynamical system induces an exchange transformation of two intervals on a cross-section perpendicular to this family. The criterion for aperiodicity of this permutation is applied later.

In the proof of Theorem 4 there appears a homologous equation which has a "good" solution, allowing us to pass from the plane into space.

Note, besides, that with the help of the construction given in the proofs of Theorems 1 and 2, we can prove the existence of periodic billiard trajectories in configuration space $Q$, approximating as closely as desired the non-periodic and not everywhere dense billiard trajectory $\Gamma$ from Theorem 1.

Denote the closure of the trajectory $\Gamma$ as $\mathbb{5} \subset Q$, and the interior of $\mathbb{5}$ as $\Omega=\operatorname{int}(\mathfrak{G}$, this being an open region in $Q$.

Call a point $q \in Q$ of configuration space $Q$ a periodic point if a periodic trajectory passes through it.

Theorem 5. The set of periodic points in $Q$ is everywhere dense in the region $\Omega$. More precisely: for any $\varepsilon>0$ and for any point $q_{0} \in Q$, there exists a point $q$ in the $\varepsilon$-neighborhood of $q_{0}$ such that a periodic billiard trajectory passes through $q$ at an angle less than $\varepsilon$ to the trajectory $\Gamma$.

Theorem 5 thus states that the billiard which generates trajectory $\Gamma$ in the polygon $Q$ can be shifted from $\Gamma$ by less than (an arbitrary) $\varepsilon$ both in its coordinate $\left(\left|q-q^{\prime}\right|<\varepsilon\right)$ and its angle $\left(\left|v-v^{\prime}\right|<\varepsilon\right)$, after which it will generate a periodic trajectory in $Q$. Besides the ideas stated above, we use the following concept in the proof of this theorem: every periodic trajectory has a bundle of parallel periodic trajectories reflecting from the same sides of the polygon as the original periodic trajectory does. 


\section{Proof of Theorem 1 for $n \geqq 4$}

Generally speaking, the assertion of Theorem 1 for $n \geqq 4$ can be obtained as a corollary of Theorem 2. Indeed, it follows from the proof of Theorem 2 that the constructed billiard trajectory never falls into a certain neighborhood of the acute angle vertex of a right triangle. Hence, that neighborhood can be replaced by another, polygonal one, forming a convex $n$-agon with a preassigned $n \geqq 4$ (Fig. 1 ).

However, because of the complexity of the proof of Theorem 2, we will give an individual proof of Theorem 1 for $n \geqq 4$, without using Theorem 2 . The case $n=3$ will be examined in the proof of Theorem 2 .

We now describe the plan of the proof. First we construct a special convex hexagon $A B C A^{\prime} B^{\prime} C^{\prime}$, in which we will afterwards construct an everywhere dense billiard trajectory which will never reflect from sides $A B$ and $A^{\prime} B^{\prime}$. Afterwards, extending sides $A C^{\prime}$ and $B C$ to their intersection, and sides $B^{\prime} C^{\prime}$ and $A^{\prime} C$ to theirs, we obtain a convex quadrangle in which the billiard trajectory will be non-periodic and not everywhere dense: it does not enter the two triangular regions added to the hexagon. If we replace one of the triangular regions by a polygon, we obtain a convex polygon with any preassigned number of sides $n>4$.

Hexagon $A B C A^{\prime} B^{\prime} C^{\prime}$ will satisfy the following conditions:

1. $A B C A^{\prime} B^{\prime} C^{\prime}$ is centrally symmetric: $A B\left\|A^{\prime} B^{\prime}, A C^{\prime}\right\| A^{\prime} C$ and $B C \| B^{\prime} C^{\prime}$ (we will consider sides $A B$ and $A^{\prime} B^{\prime}$ as vertical); (Fig. 2);

2. The angle at vertex $A$ equals $\frac{\pi}{2}+\alpha$ and at vertex $B$ equals $\frac{\pi}{2}+\beta$ $\left(0<\alpha, \beta<\frac{\pi}{2}\right)$, where

$$
\cot 2 \alpha>\tan \beta, \quad \cot 2 \beta>\tan \alpha .
$$

3. The perpendicular at point $A$ to side $A C^{\prime}$ is the bisector of angle $B A C$ and the perpendicular at point $B$ to side $B C$ is the bisector of angle $A B C^{\prime}$ (Fig. 2).

Fig. 1
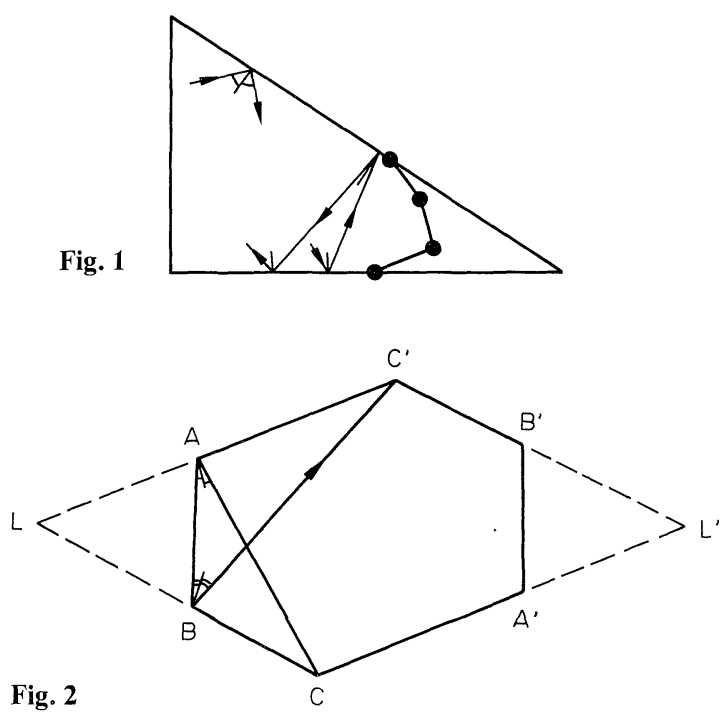
The existence of a hexagon satisfying conditions 1.-3. is seen from the following. Consider segment $A B$ and draw straight lines $B C^{\prime}$. and $A C$ at angles $2 \alpha$ and $2 \beta$, intersecting by virtue of inequalities (1) (these inequalities will naturally appear further on, and we will not discuss them now).

Next, by drawing lines through points $A$ and $B$, perpendicular to the bisectors of the angles $B A C$ and $A B C^{\prime}$, respectively, we find the points $C$ and $C^{\prime}$ at their intersections with the straight lines $A C$ and $B C^{\prime}$, respectively. Then we complete the figure obtained to form a centrally symmetric hexagon $A B C A^{\prime} B^{\prime} C^{\prime}$.

Let us clarify the meaning of condition 3 . If we start a particle from point $B$ to point $A$ (forget for a while that $A$ and $B$ are vertices), after reflecting from the straight line $A C^{\prime}$, the particle falls into point $C$; if it is started from point $A$ to point $B$, then after reflecting from the straight line $B C$ it falls into point $C^{\prime}$. This behavior of the particle gives us the desired trajectory.

We will call the segment between two neighboring reflections of the billiard trajectory from boundary $\partial Q$ a link of the billiard trajectory in $Q$. Transformation $S$, which turns every trajectory link into the following one, acts on the set of billiard trajectory links; we will say that the trajectory link reflects from the boundary under the action of $S$.

Consider the family $\mathfrak{U}$ of segments parallel to the side $A B$ of the hexagon, the ends of which lie on the hexagon's sides (vertical family). Every segment $\gamma \in \mathfrak{A}$ may be considered as a link of some billiard trajectory in a hexagon; we will also call $\mathfrak{A}$ a family of vertical links. Mentally divide this family into two families : the first (I) contains all the segments between side $A B$ and segment $\kappa$, which passes through vertex $C^{\prime}$ parallel to $A B$; the second (II) consists of all the segments between $\kappa$ and side $A^{\prime} B^{\prime}$.

All the segments of family I reflect from side $A C^{\prime}$ under the action of $S$, and all segments of family II reflect from side $B^{\prime} C^{\prime}$. After reflecting, family I turns into a family of segments parallel to $A C$, and family II turns into a family of segments parallel to $B C^{\prime}$ (Fig. 3). Then these reflected families reflect from sides $A^{\prime} C$ and $B C$, symmetric to sides $A C^{\prime}$ and $B^{\prime} C^{\prime}$, and uniting, turn into a family of vertical segments again.

Hence, if some link $\gamma$ of the billiard trajectory belongs to family $\mathfrak{A}$ (vertical), every second link of this trajectory also belongs to $\mathfrak{A}$.

For a vertical link $\gamma$ of the billiard trajectory, denote by $F(\gamma)$ the first vertical link that $\gamma$ turns into after reflecting from a pair of opposite sides of the hexagon. The mapping of family $\mathfrak{A}$ into itself is thus defined: $F: \mathfrak{U} \rightarrow \mathfrak{U} ; F$ is the square of transformation $S$.

Fig. 3

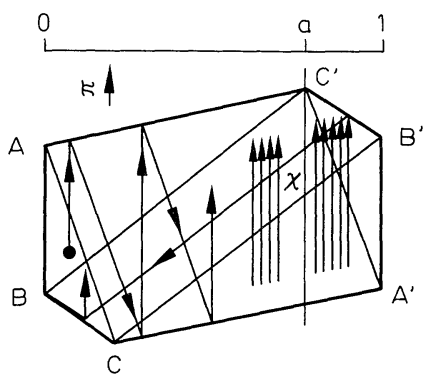


Let $\pi$ be the orthogonal projection of the vertical links of family $\mathfrak{A}$ onto a horizontal straight line, and let $A B$ project into the endpoint $\{0\}$, and $A^{\prime} B^{\prime}$ into the endpoint $\{1\}$ of the interval $[0,1]$ (Fig. 3). Then under projection $\pi$, the image of family I is an interval $[0, a] \subset[0,1]$. Denote the length of interval $[a, 1]$ as $b=1-a$. The mapping $F: \mathfrak{A} \rightarrow \mathfrak{U}$ induces a mapping $f$ of the interval $[0,1]$ into itself, $f$ being defined by $F$ according to the formula

$$
f(x) \stackrel{\text { def }}{=} \pi(F(\gamma)), \text { where } x \in[0,1] \text { and } x=\pi(\gamma) .
$$

Using property 3. of the hexagon $A B C A^{\prime} B^{\prime} C^{\prime}$, we can rewrite equality (2) as

$$
f(x)=\left\{\begin{array}{lll}
x+b & \text { if } & x \leqq a \\
x-a & \text { if } & x \geqq a
\end{array}\right.
$$

or, in short, as

$$
f(x)=(x+b) \bmod 1 .
$$

Formula (4) defines the exchange transformation of the intervals $[0, a]$ and $[a, 1]$.

As is known, for the exchange transformation of two intervals, the trajectory of any point $x \in[0,1]$ is everywhere dense on $[0,1]$ if, and only if, the length ratio $\frac{a}{b}$ of these intervals is irrational (otherwise the trajectory is periodic). Hence, the billiard trajectory, the first link of which belongs to family $\mathfrak{A}$, is everywhere dense in hexagon $A B C A^{\prime} B^{\prime} C^{\prime}$ if, and only if, $\frac{a}{b} \notin \mathbb{Q}$.

We will express this ratio in terms of the angles $\alpha$ and $\beta$ (Fig. 4). From right triangles $A C^{\prime} M, B C^{\prime} N$, and $A C K$, in which $A M=B N=a$ and $C K=b$, we obtain

$$
a=A B \cdot(\cot 2 \beta-\tan \alpha)^{-1}, \quad b=A B \cdot(\cot 2 \alpha-\tan \beta)^{-1}
$$

[by virtue of inequalities (1), $a$ and $b$ are positive]. Hence,

$$
\frac{a}{b} \stackrel{\text { def }}{=} h(\alpha, \beta)=(\cot 2 \alpha-\tan \beta)(\cot 2 \beta-\tan \alpha)^{-1} \text {. }
$$

Now note that the function $h(\alpha, \beta)$ is continuously differentiable and nonconstant on a region produced on the plane $(\alpha, \beta)$ by inequalities (1). Therefore,

Fig. 4

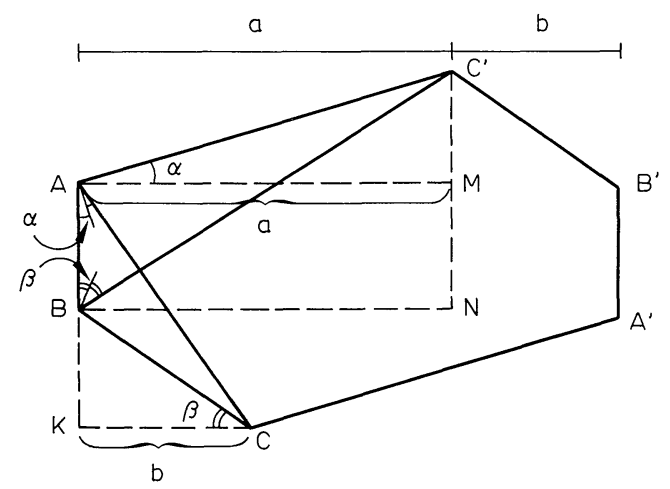


$h(\alpha, \beta) \notin \mathbb{Q}$ for almost all $(\alpha, \beta)$ of some region. Among the hexagons produced by such $\alpha$ and $\beta$, some have angles incommensurate with $\pi$. Indeed, for almost all $\alpha$ and $\beta$ satisfying condition (1), the corresponding hexagon will satisfy our requirements, having angles incommensurate with $\pi$. Then the desired $n$-agon, $n \geqq 4$, obtained from our hexagon by adding two triangular regions and clipping one or both of them, as described above, can have all angles incommensurate with $\pi$.

However, for any $n \geqq 4$ there exists a convex rational $n$-agon in which there is a non-periodic billiard trajectory that is not everywhere dense. To prove the existence of such an $n$-agon we will present a concrete example.

First we will construct a rational hexagon $A B C A^{\prime} B^{\prime} C^{\prime}$ with properties 1.-3., in which the trajectory of the type described above is everywhere dense.

Assume that $\alpha=\frac{\pi}{12}, \beta=\frac{\pi}{6}$; then the angles at the hexagon's vertices $A, B$, and $C$ equal $\frac{7 \pi}{12}, \frac{2 \pi}{3}$, and $\frac{3 \pi}{4}$, respectively. The value of the function $h(\alpha, \beta)$ for these $\alpha$ and $\beta$ equals

$$
h\left(\frac{\pi}{12}, \frac{\pi}{6}\right)=\left(\sqrt{3}-\frac{1}{\sqrt{3}}\right)\left(\frac{1}{\sqrt{3}}-\frac{1}{2+\sqrt{3}}\right)^{-1}=2+\sqrt{3} \notin \mathbb{Q},
$$

and therefore the billiard trajectory is everywhere dense in the hexagon.

It follows that for any $n$-agon, $n>4$, obtained from this hexagon by the method described above, the angle at vertices $C$ and $C^{\prime}$ equals $\frac{3 \pi}{4}$, and we can choose all the remaining angles to be commensurate with $\pi$. For $n=4$ there is no such freedom of choice, but by extending sides $A C^{\prime}$ and $B C$, and also $B^{\prime} C^{\prime}$ and $A^{\prime} C$ to

their intersections, we obtain the parallelogram $L C L^{\prime} C^{\prime}$ with acute angle $\frac{\pi}{4}$ (Fig. 2).
Thus Theorem 1 is proved for $n \geqq 4$. $\square$

Remark. As was mentioned in the Introduction, in rational polygons the billiard problem is reduced to windings on closed oriented two-dimensional manifolds. Every one of these manifolds is obtaining by sewing together [i.e., identifying the sides according to formula $(*)$ of the Introduction] a number of copies of the original polygon $Q$; the genus of this manifold is determined only by the type of the polygon (see Statement 1, [1]). Several branching points of the parallel billiard flow arise on this manifold: they are obtained by identifying the vertices of the polygon whose angles have values $\pi \frac{m}{n}, m>1$; the number $m$ is called the order of branching. In the neighborhood of any point of the manifold, except for the branching points, the billiard family induced on the manifold is a local family of parallel shifts (or in the neighborhood of vertices of $\frac{\pi}{n}$-valued angles the family is
supplemented to a continuous flow).

For this family, the branching point of order $m$ is a multisaddle of order $1-m$, i.e., the phase portrait of the family in the neighborhood of this point consists of $2 m$ hyperbolic sectors separated by $m$ in- and $m$ out-separatrices (see Statement 2, [1]). 


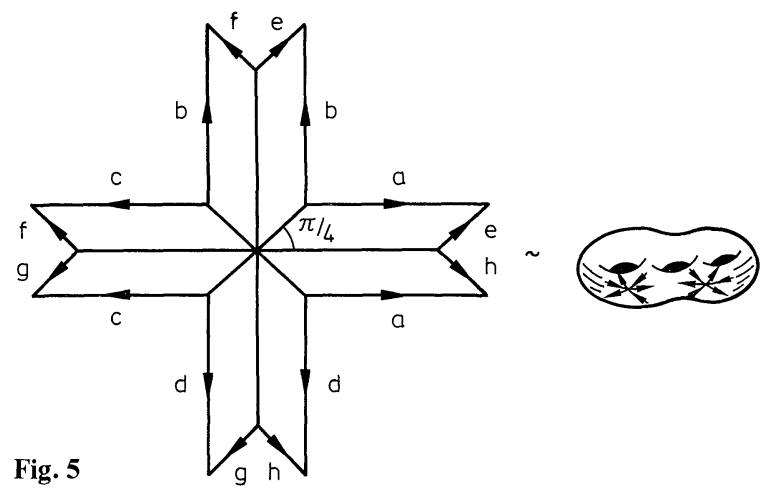

From this, by Euler's formula it is easy to calculate the genus of the manifold using the polygon's angles $\pi \frac{m_{i}}{n_{i}}$ :

$$
2-2 g=\sum_{i}\left(1-m_{i}\right) \Rightarrow g=1+\frac{1}{2} \sum_{i}\left(m_{i}-1\right) .
$$

We will describe the topological behavior on an invariant manifold of a nonperiodic and not everywhere dense billiard trajectory constructed in a paralelogram with angle $\frac{\pi}{4}$. By reflecting the parallelogram from its sides adjoining the vertex of angle $\frac{\pi}{4}$, we obtain a non-convex 16-agon, the sides of which are identified as shown in Fig. 5. After identifying the corresponding sides, it becomes a surface $\Lambda$ of genus $g=1+\frac{1}{2}(2+2)=3$, divided into eight curvilinear parallelograms (for this and analogous examples, the genus can be calculated, as is known in twodimensional topology, by selecting cells of type $a b a^{-1} b^{-1}$ in the "word" $w$ defining the polygon). There are two branching points, both of them of order 3 .

It is possible to trace, in detail the behavior of the family of parallel segments $\mathfrak{A}$ (in the hexagon $A B C A^{\prime} B^{\prime} C^{\prime}$ ) on the surface $A$. To do this, we must draw a set of patterns and identify (sew) the corresponding sides; this is cumbersome, so we will not consider it here.

It is simpler to do as follows: Since there is an exchange transformation of the two intervals, we shall separately trace I, the left family of parallel segments, and II, the right family, in the union of segments of family $\mathfrak{A}$, whose cross-section is $[0,1]$.

Family I defines the left "strip," obtained by the movement of segment $[0, a]$ until it lands in segment $[1-a, 1]$; similarly, family II defines the right "strip," obtained by the movement of segment $[a, 1]$ until it lands in segment $[0,1-a]$. Both strips should be imagined as being located in $\mathbb{R}^{3}$; then there appears the surface presented in the left part of Fig. 6. This surface is homeomorphic to a twodimensional torus Tor $^{2}$ with a hole, shown in the right part of Fig. 6 . The billiard trajectory on Tor $^{2}$ which flows past the hole and winds around the remaining part of Tor ${ }^{2}$, and which is everywhere dense, is also shown on Fig. 6. In particular, both branching points are shown; two separatrices enter and one exits from one of the 
Fig. 6

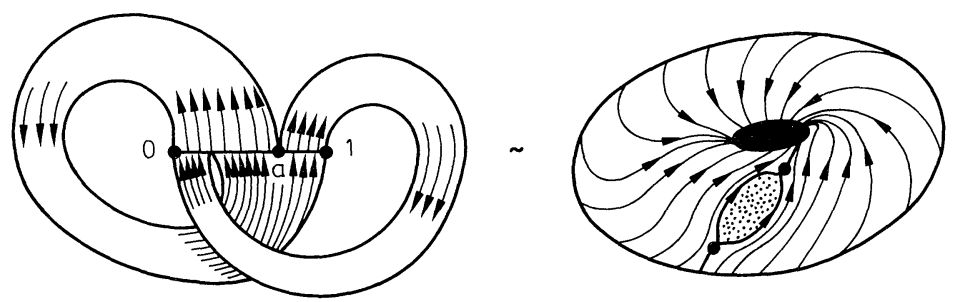

points, and two separatrices exit and one enters the other point (Fig. 6). Note that the billiard trajectories on Tor $^{2}$ with vertical links in family $\mathfrak{A}$ separate into trajectories to the left and to the right of the hole and then merge. This accords with the situation described.

In order to present the billiard trajectories with links of family $\mathfrak{U}$ on the whole manifold $\Lambda$ we must cover up the hole in Tor $^{2}$ with a disk and two handles. The handles, as well as the interior of the disk, are free from billiard trajectories (the two remaining triplets of separatrices are located on them).

The same topological situation takes place in the general case when examining the billiard family with links in $\mathfrak{A}$, on the cross-section of which two segments are exchanged in any rational polygon. If the manifold $\Lambda$ of genus $g$ corresponds to this polygon, then $\Lambda$ can be represented as the union of Tor ${ }^{2}$ and the $(g-1)$ handles attached to the Tor $^{2}$ on the disk. The billiard trajectory corresponding to the interval exchange transformation indicated lies on $\left(\operatorname{Tor}^{2} \backslash\right.$ disk) and acts as in Fig. 6.

\section{Proof of Theorem 2. Corollary: Proof of Theorem 1 for $n=3$}

As in Sect. 2 , in the case of a triangle $(n=3)$ we will use the same construction: an exchange transformation of two segments on the cross-section of the family of parallel billiard trajectories.

\subsection{Reducing the Billiard Flow to an Exchange Transformation of Two Segments}

We will fix a right triangle with acute angle $\alpha$. By reflecting it from the side opposite angle $\alpha$, we obtain an isosceles triangle $A B C$ with angle $\pi-2 \alpha$ at vertex $C$. After the reflection, a billiard trajectory in the right triangle will become a billiard trajectory in the triangle $A B C$. Triangle $A B C$ will be the basic one in what follows. Vertex $C$ will simply be the vertex and side $A B$ will be the base.

Consider a family $\mathfrak{A}$ of parallel segments, not parallel to the base, in triangle $A B C$. Distinguish particularly the segment of the family, which passes through vertex $C$ (Fig. $7 \mathrm{a} ; \jmath=C S$ ). Assume that the point $S$, the endpoint of segment $\delta$ on base $A B$, is located near the middle of $A B$. Trace any two billiard trajectories in the triangle, the initial links of which a) belong to family $\mathfrak{A}$, b) are "infinitely close" to segment $\mathrm{s}$, and c) are located to the right and to the left of $\mathrm{s}$. We will call these trajectories the right and the left, respectively. 

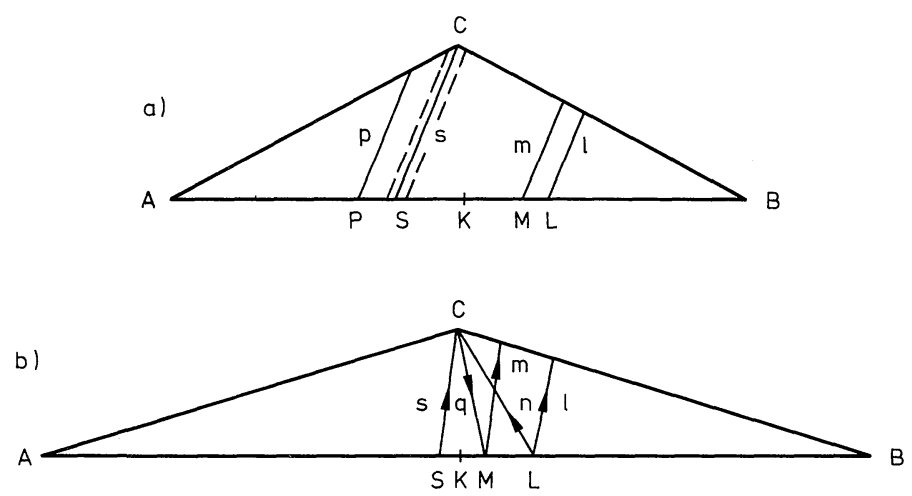

Fig. $7 \mathrm{a}$ and $b$

Assume that after many reflections, some link $\ell$ of the left trajectory is situated to the right of segment $s$ and parallel to it, and that some link $\not$ of the right trajectory is situated to the left of $s$ and also parallel to it. Then we can hope that the bundle of segments of family $\mathfrak{A}$ which is located between $\not h$ and $s$ (the left bundle), will reflect from the sides of triangle $A B C$ in the same way as the left trajectory reflects, and that after some reflections it will turn into a bundle of segments parallel to $\delta$, located between some segment $m(\| \jmath)$ and segment $\ell$; similarly, the bundle of parallel segments between $s$ and $\ell$ (the right bundle) will turn into a bundle of parallel segments between $\not$ and $m$ (Fig. 7a). In this case, segments $P S$ and $S L$ will be exchanged on the segment $P L$, which is located on the base $A B: P S \rightarrow M L, S L \rightarrow P M$.

To fulfil this hope, the following is necessary: In the billiard trajectory which contains segment $m$ as a link, the link directly preceding $m$ should exit from vertex $C$ into point $M$ (i.e., the particle should reflect from side $A C$ onto point $C$ and should fall into point $M$ on $A B$. Thus, this billiard trajectory is degenerate).

Indeed, in our case, the left and right bundles moved independently to point $C$, but after some reflections from the triangle's sides they went parallel to the mutual link $m$, reflecting from the base $A B$ immediately before that. This means that the preceding links of these bundles' trajectories (directly before their reflection from $A B$ ) were also parallel among themselves. However, one part of these preceding links reflected from side $B C$ and the other from side $A C$. From this it follows that after reflecting from the lateral sides, the bundles went parallel to each other along the mutual link $C M$, which lands in link $m$ after reflecting from the base $A B$. In other words, the left trajectory's evolution (if we continue it beyond link $\ell$ ) must have the following form: $s \ldots \ell \ldots n q m$ (Fig. 7b). Here link $n$ lands in link $q$ after it reflects from side $A C$ at vertex $C$, and segments $s$ and $\phi$ are symmetric in the segment $C K$.

\subsection{Straightening the Trajectory}

We will represent the desired trajectory in straightened form, using the known procedure for straightening a billiard trajectory in an arbitrary polygon. A 


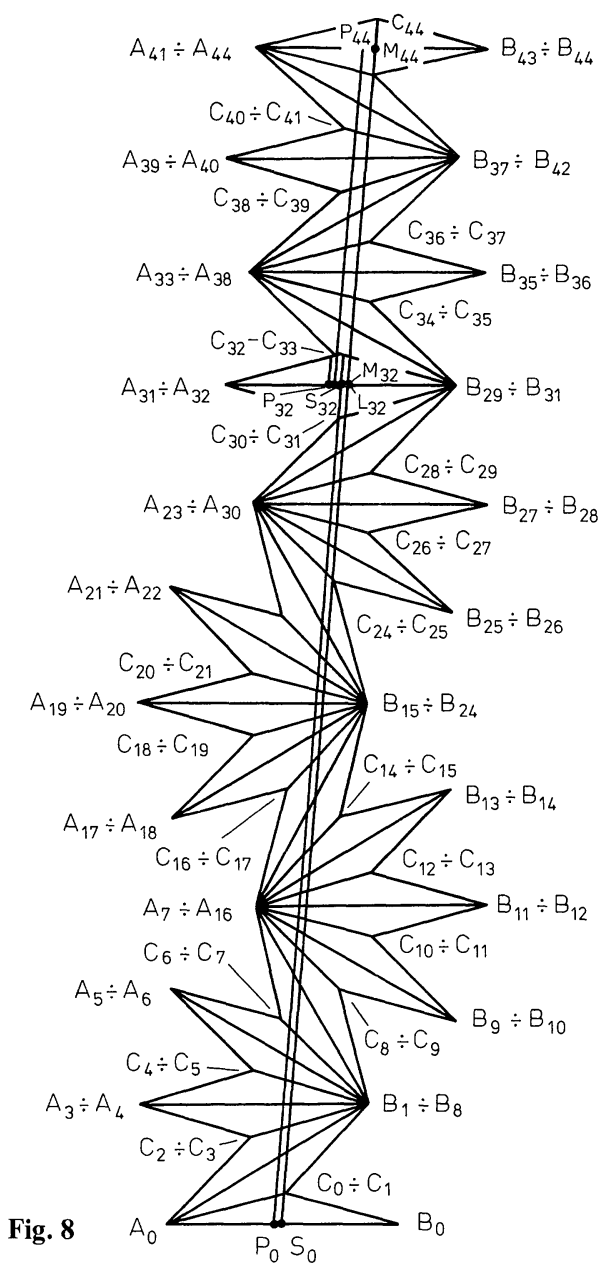

straightened trajectory which satisfies the conditions of the previous section is shown in Fig. 8.

In the process of straightening the billiard trajectory, there appear 45 triangles obtained by reflections of triangle $A B C$ from its sides. Number these triangles $0,1, \ldots, 44(0$ corresponds to triangle $A B C)$. We will use a single letter with an index to designate any point's image obtained by reflections of triangle $A B C$, the index indicating the triangle's number. In particular, triangle $A_{0} B_{0} C_{0}$ is triangle $A B C$; all points without indices belong to triangle $A B C$. We will call points $C_{0}=C, C_{1}, \ldots, C_{44}$ the vertices of the reflected triangles.

The straightened trajectory connects vertices of the $0^{\text {th }}$ and the $43^{\text {rd }}$ triangles (points $C_{0}$ and $C_{43}$ ) and intersects the base of triangle No. 32 at point $L_{32}$, the base of triangle No. 0 at point $S_{0}=S$, and the base of triangle No. 44 at point $M_{44}$. The "corridor" between the parallel segments $S_{0} L_{32}$ and $P_{0} M_{32}$ (the latter passing through vertex $C_{31}$; triangle $A_{31} B_{31} C_{31}$ is upside down and side $A_{31} B_{31}$ is horizontal) shows the mapping of the bundle of parallel segments between links $\not h$ 
and $s$ from Fig. 7a into a bundle of parallel segments between links $m$ and $\ell$. Analogously, the corridor between segments $L_{32} M_{44}$ and $S_{32} P_{44}$ (the latter passing through vertex $C_{32}$ ) shows the mapping of the bundle of segments between links $\sigma$ and $\ell$ into a bundle of segments between links $\not \rho$ and $m$.

To prove that this construction can be realized, we must check that the corridor indicated intersects precisely the sides of the triangle as shown in Fig. 8; for instance, vertex $B_{1}$ lies to the right of the corridor, vertex $C_{6}$ to the left, and so on. To do this, we must make complicated computations (we omit them here), showing that with a sufficiently small $\alpha$, such geometric correlations do take place. The idea of these computations can be understood from the computations of certain vertex coordinates, as follows.

\subsection{Linear Transformations of Rhombuses}

We shall prove that the ratio of lengths of the transmuted segments PS and SL is irrational. We shall calculate the coordinates of the points we are interested in. We will place the origin of coordinates in the middle $K$ of segment $A B$, direct axis $O X$ along the beam $K B$, and axis $O Y$ perpendicular to the axis $O X$ along the beam $K C$.

Instead of considering triangles $\left\{A_{i} B_{i} C_{i}\right\}$, we will paste them in pairs and consider the rhombuses obtained. We will designate the rhombus obtained by pasting triangles with numbers $k$ and $k+1, k \geqq 1$, as $Q_{k, k+1}$. Moreover, we will reflect triangle No. 0 from the base $A B$ and designate the rhombus obtained as $Q_{-1,0}$.

In order to find the coordinates of the vertices in a triangle we will define an affine transformation of the plane that transforms rhombus $Q_{-1,0}$ into the rhombus we are interested in. We will construct the affine transformation as a composition of elementary transformations $\mathbf{L}$ and $\mathbf{R}$ :

$\mathbf{L}=R_{A}^{\varphi}$ is the rotation of the plane around vertex $A$ counter-clockwise by angle $\varphi=2 \alpha$, equal to the angle at the rhombus vertex (the transformation $\mathbf{L}$ turns rhombus $Q_{-1,0}$ into rhombus $Q_{1,2}$ );

$\mathbf{R}=R_{B}^{-\varphi}$ is the rotation of the plane around vertex $B$ clockwise by angle $\varphi=2 \alpha$.

Moreover, denote parallel transport along vector $\mathbf{x}$ by $\mathbf{T}_{\mathbf{x}}$. Assume the length of segment $A B$ is one. We will write the result of applying any transformation $\mathscr{A}$ to the argument $\xi$ as $\xi \mathscr{A} ; \xi \mathscr{A} \mathscr{B}$ means $(\xi \mathscr{A}) \mathscr{B}$.

Any rhombus $Q_{k, k+1}$ can be obtained from the rhombus $Q_{-1,0}$ by a sequence of transformations $\mathbf{L}$ and $\mathbf{R}$. Construct this sequence as follows: Move along the chain of rhombuses from rhombus $Q_{k, k+1}$ to rhombus $Q_{-1,0}$, and for every pair of neighboring rhombuses in this chain write the letter $\mathbf{L}$ or $\mathbf{R}$ depending on whether the rhombuses have a mutual left vertex (i.e., vertex $A_{r}, r$ being an index), or a mutual right vertex (i.e., vertex $B_{r}$ ). For instance (Fig. 8),

$$
\begin{aligned}
Q_{43,44} & =Q_{-1,0} \text { LRRLLRRLLLRRRRLLLLRRRL } \\
& =Q_{-1,0} \mathbf{L R}^{2} \mathbf{L}^{2} \mathbf{R}^{2} \mathbf{L}^{3} \mathbf{R}^{4} \mathbf{L}^{4} \mathbf{R}^{3} \mathbf{L}
\end{aligned}
$$

We will need Formula (6) further on.

4 In order to prove the assertion, denote the transformation turning rhombus $Q_{k, k+1}$ into rhombus $Q_{m, m+1}$ by $\mathscr{A}_{k, m}$ (in particular, $Q_{-1,0} \mathscr{A}_{-1, m}=Q_{m, m+1}$ ). We 
want to represent transformation $\mathscr{A}_{-1, m}$ as a composition of transformations $\mathbf{R}$ and $\mathbf{L}$. First, we find the connection between the transformations $\mathscr{A}_{m, m+2}$ and $\mathscr{A}_{-1, m}$.

Transformation $\mathscr{A}_{m, m+2}$ turns rhombus $Q_{m, m+1}$ into rhombus $Q_{m+1, m+2}$. This mapping can be represented thus: first turn rhombus $Q_{m, m+1}$ into rhombus $Q_{-1,0}$ by the transformation $\mathscr{A}_{-1, m}^{-1}$, then make an $\mathbf{L}$ or $\mathbf{R}$ rotation of rhombus $Q_{-1,0}$ (depending on which rotation, left or right, turns rhombus $Q_{m, m+1}$ into $Q_{m+1, m+2}$ ), and then turn rhombus $Q_{-1,0}$ into $Q_{m, m+1}$ by the transformation $\mathscr{A}_{-1, m}$; the new rhombus will be $Q_{m+1, m+2}$. Thus,

$$
\mathscr{A}_{m, m+2}=\mathscr{A}_{-1, m}^{-1}\left(\begin{array}{l}
\mathbf{L} \\
\mathbf{R}
\end{array}\right)_{m, m+2} \mathscr{A}_{-1, m} .
$$

From this, the recurrence relation

$$
\mathscr{A}_{-1, m+2}=\mathscr{A}_{-1, m} \mathscr{A}_{m, m+2}=\mathscr{A}_{-1, m} \mathscr{A}_{-1, m}^{-1}\left(\begin{array}{l}
\mathbf{L} \\
\mathbf{R}
\end{array}\right)_{m, m+2} \mathscr{A}_{-1, m}=\left(\begin{array}{l}
\mathbf{L} \\
\mathbf{R}
\end{array}\right)_{m, m+2} \mathscr{A}_{-1, m}
$$

follows, and from the latter we find that for any $r$

$$
\mathscr{A}_{-1, r}=\left(\begin{array}{l}
\mathbf{L} \\
\mathbf{R}
\end{array}\right)_{r-2, r}\left(\begin{array}{l}
\mathbf{L} \\
\mathbf{R}
\end{array}\right)_{r-4, r-2} \ldots\left(\begin{array}{l}
\mathbf{L} \\
\mathbf{R}
\end{array}\right)_{-1,1},
$$

since $\mathscr{A}_{-1,1}$ is the identity transformation.

Thus, the assertion stated has been proved: The chain of transformation $\mathbf{L}$ and $\mathbf{R}$ should be written as described above.

\subsection{Calculations}

In order to compute the compositions of the rotations $\mathbf{L}$ and $\mathbf{R}$ obtained, use the identities

$$
R_{A}^{\beta} R_{B}^{-\beta}=\mathbf{T}_{\mathbf{v}_{\beta}}, \quad R_{B}^{-\beta} R_{A}^{\beta}=\mathbf{T}_{\mathbf{u}_{\beta}},
$$

where

$$
\mathbf{v}_{\beta}=(1-\cos \beta, \sin \beta), \quad \mathbf{u}_{\beta}=(-1+\cos \beta, \sin \beta) .
$$

For an arbitrary point $H$ of the plane and a vector $\mathbf{x}$,

$$
R_{H}^{\beta} \mathbf{T}_{\mathbf{x}}=\mathbf{T}_{\mathbf{x} R^{-\beta}} R_{H}^{\beta}, \quad \mathbf{T}_{\mathbf{x}} R_{H}^{\beta}=R_{H}^{\beta} \mathbf{T}_{\mathbf{x} R^{\beta}},
$$

where $R^{\beta}$ is the rotation of linear two-dimensional space $\mathbb{R}^{2}$ by angle $\beta$. Note that the transformations $\mathbf{L}$ and $\mathbf{R}$ introduced are just the transformations $R_{A}^{\varphi}$ and $R_{B}^{-\varphi}$ :

$$
\mathbf{L}=R_{A}^{\varphi}, \mathbf{R}=R_{B}^{-\varphi} .
$$

Simplify formula (6) with the help of identities (7) and (9):

$$
\begin{aligned}
\mathbf{L} \mathbf{R}^{2} \mathbf{L}^{2} \mathbf{R}^{2} \mathbf{L}^{3} \mathbf{R}^{4} \mathbf{L}^{4} \mathbf{R}^{3} \mathbf{L} & =\mathbf{L}\left(\mathbf{R}^{2} \mathbf{L}^{2}\right)\left(\mathbf{R}^{2} \mathbf{L}^{2}\right) \mathbf{L}\left(\mathbf{R}^{4} \mathbf{L}^{4}\right) \mathbf{R}^{2}(\mathbf{R} \mathbf{L}) \\
& =\mathbf{L} \mathbf{T}_{\mathbf{u}_{2 \varphi}} \mathbf{T}_{\mathbf{u}_{2 \varphi}} \mathbf{L} \mathbf{T}_{\mathbf{u}_{4 \varphi}} \mathbf{R}^{2}=\mathbf{L} \mathbf{L} \mathbf{T}_{\left(2 \mathbf{u}_{2 \varphi} R^{\varphi}+\mathbf{u}_{4 \varphi}\right)} \mathbf{R}^{2} \mathbf{T}_{\mathbf{u}_{\varphi}} \\
& =\mathbf{L}^{2} \mathbf{R}^{2} \mathbf{T}_{\left(2 \mathbf{u}_{2 \varphi} R^{\varphi}+\mathbf{u}_{4 \varphi}\right) R^{-2 \varphi}+\mathbf{u}_{\varphi}}=\mathbf{T}_{\left(\mathbf{v}_{2 \varphi}+2 \mathbf{u}_{2 \varphi} R^{-\varphi}+\mathbf{u}_{4 \varphi} R^{-2 \varphi}+\mathbf{u}_{\varphi}\right)} .
\end{aligned}
$$


Thus, transformation (10), which turns $Q_{-1,0}$ into $Q_{43,44}$ and hence vertex $C_{-1}$ into vertex $C_{43}$, is a parallel transfer along the vector (Fig. 8)

$$
\overrightarrow{C_{-1} C_{43}}=\mathbf{v}_{2 \varphi}+2 \mathbf{u}_{2 \varphi} R^{-\varphi}+\mathbf{u}_{4 \varphi} R^{-2 \varphi}+\mathbf{u}_{\varphi} .
$$

From this we see that

$$
\overrightarrow{C_{0} C_{43}}=\overrightarrow{C_{-1} C_{43}}-\overrightarrow{C_{-1} C_{0}}=\mathbf{v}_{2 \varphi}+2 \mathbf{u}_{2 \varphi} R^{-\varphi}+\mathbf{u}_{4 \varphi} R^{-2 \varphi}+\mathbf{u}_{\varphi}-\left(\begin{array}{c}
0 \\
\tan \frac{\varphi}{2}
\end{array}\right) \text {. }
$$

We will further assume that the angle $\varphi$ is small and we will make a Taylor series expansion about the point $\varphi=0$ for all the functions with argument $\varphi$. Each time it will be sufficient for the proof to consider only the first several terms and to bound the remaining ones by $O\left(\varphi^{n}\right)$ with an appropriate $n$.

For further calculation we will need the vectors $\mathbf{v}_{\varphi}, \mathbf{u}_{\varphi}$ in coordinate form and the linear transformations $R^{\varphi}, R^{-\varphi}$ in matrix form.

$$
\begin{aligned}
& \left.\begin{array}{l}
\mathbf{v}_{\varphi}=(1-\cos \varphi, \sin \varphi)=\left(\frac{1}{2} \varphi^{2}-\frac{1}{24} \varphi^{4}+O\left(\varphi^{6}\right), \varphi-\frac{1}{6} \varphi^{3}+O\left(\varphi^{5}\right)\right) \\
\mathbf{u}_{\varphi}=(-1+\cos \varphi, \sin \varphi)=\left(-\frac{1}{2} \varphi^{2}+\frac{1}{24} \varphi^{4}+O\left(\varphi^{6}\right), \varphi-\frac{1}{6} \varphi^{3}+O\left(\varphi^{5}\right)\right),
\end{array}\right\} \\
& R^{\varphi}=\left(\begin{array}{cc}
\cos \varphi & -\sin \varphi \\
\sin \varphi & \cos \varphi
\end{array}\right)=\left(\begin{array}{cc}
1-\frac{1}{2} \varphi^{2}+\frac{1}{24} \varphi^{4}+O\left(\varphi^{6}\right), & -\varphi+\frac{1}{6} \varphi^{3}+O\left(\varphi^{5}\right) \\
\varphi-\frac{1}{6} \varphi^{3}+O\left(\varphi^{5}\right), & 1-\frac{1}{2} \varphi^{2}+\frac{1}{24} \varphi^{4}+O\left(\varphi^{6}\right)
\end{array}\right) \\
& \left.R^{-\varphi}=\left(\begin{array}{cc}
\cos \varphi & \sin \varphi \\
-\sin \varphi & \cos \varphi
\end{array}\right)=\left(\begin{array}{cc}
1-\frac{1}{2} \varphi^{2}+\frac{1}{24} \varphi^{4}+O\left(\varphi^{6}\right), & \varphi-\frac{1}{6} \varphi^{3}+O\left(\varphi^{5}\right) \\
-\varphi+\frac{1}{6} \varphi^{3}+O\left(\varphi^{5}\right), & 1-\frac{1}{2} \varphi^{2}+\frac{1}{24} \varphi^{4}+O\left(\varphi^{6}\right)
\end{array}\right)\right\} .
\end{aligned}
$$

Now we change the right hand side of (12) with the help of formulas (13) and (14), writing the first three summands separately.

$$
\begin{aligned}
& \mathbf{v}_{2 \varphi}=\left(2 \varphi^{2}-\frac{2}{3} \varphi^{4}+O\left(\varphi^{6}\right), 2 \varphi-\frac{4}{3} \varphi^{3}+O\left(\varphi^{5}\right)\right) ; \\
2 \mathbf{u}_{2 \varphi} R^{-\varphi}=2 & \cdot\left(\begin{array}{cc}
1-\frac{1}{2} \varphi^{2}+\frac{1}{24} \varphi^{4}+O\left(\varphi^{6}\right), & \varphi-\frac{1}{6} \varphi^{3}+O\left(\varphi^{5}\right) \\
-\varphi+\frac{1}{6} \varphi^{3}+O\left(\varphi^{5}\right), & 1-\frac{1}{2} \varphi^{2}+\frac{1}{24} \varphi^{4}+O\left(\varphi^{6}\right)
\end{array}\right) \\
& \cdot\left(\begin{array}{cc}
-2 \varphi^{2}+\frac{\varphi^{4}}{6}+O\left(\varphi^{6}\right) \\
2 \varphi-\frac{4 \varphi^{3}}{3}+O\left(\varphi^{5}\right)
\end{array}\right) \\
\mathbf{u}_{4 \varphi} R^{-2 \varphi}= & \left(\begin{array}{cc}
1-2 \varphi^{2}+\frac{2}{3} \varphi^{4}+O\left(\varphi^{6}\right), \\
-2 \varphi+\frac{4}{3} \varphi^{3}+O\left(\varphi^{5}\right), & \left.1-2 \varphi^{6}\right), 4 \varphi-\frac{2}{3} \varphi^{3}+O\left(\varphi^{5}\right) \\
-2 &
\end{array}\right) \\
& \cdot\left(\begin{array}{c}
-8 \varphi^{4}+O\left(\varphi^{6}\right) \\
-8 \varphi^{2}+\frac{16}{3} \varphi^{4}+O\left(\varphi^{6}\right) \\
4 \varphi-\frac{16}{3} \varphi^{3}+O\left(\varphi^{5}\right)
\end{array}\right) \\
= & \left(\frac{16}{3} \varphi^{4}+O\left(\varphi^{6}\right), 4 \varphi+\frac{8}{3} \varphi^{3}+O\left(\varphi^{5}\right)\right) .
\end{aligned}
$$


In coordinate form, Formula (12) is rewritten thus:

$$
\overrightarrow{C_{0} C_{43}}=\left(\frac{3}{2} \varphi^{2}+\frac{89}{24} \varphi^{4}+O\left(\varphi^{6}\right), \frac{21}{2} \varphi+\frac{13}{24} \varphi^{3}+O\left(\varphi^{5}\right)\right) .
$$

Now write the transformation that turns rhombus $Q_{-1,0}$ into rhombus $Q_{31,32}$, using the assertion of Sect. 3.3 and also formulas (7) and (9):

$$
\begin{aligned}
Q_{31,32} & =Q_{-1,0} \mathbf{R L L L R R R R L L L L R R R L} \\
& =Q_{-1,0} \mathbf{R L}^{3} \mathbf{R}^{4} \mathbf{L}^{4} \mathbf{R}^{3} \mathbf{L}=Q_{-1,0}(\mathbf{R L}) \mathbf{L}^{2}\left(\mathbf{R}^{4} \mathbf{L}^{4}\right) \mathbf{R}^{2}(\mathbf{R L}) \\
& =Q_{-1,0} \mathbf{T}_{\mathbf{u}_{\varphi}} \mathbf{L}^{2} \mathbf{T}_{\mathbf{u}_{4 \varphi}} R^{2} \mathbf{T}_{\mathbf{u}_{\varphi}}=Q_{-1,0} \mathbf{T}_{2 \mathbf{u}_{2 \varphi}+\mathbf{u}_{4 \varphi} R^{-2 \varphi}+\mathbf{v}_{2 \varphi}} .
\end{aligned}
$$

Thus, rhombus $Q_{31,32}$ is a copy of rhombus $Q_{-1,0}$, under parallel transport along vector $\xi=\overrightarrow{B_{0} B_{32}}$ (since vertices $B_{0}$ and $B_{32}$ coincide after this transfer). From (16), we see that

$$
\xi=\overrightarrow{B_{0} B_{32}}=2 \mathbf{u}_{2 \varphi}+\mathbf{u}_{4 \varphi} R^{-2 \varphi}+\mathbf{v}_{2 \varphi} .
$$

Now calculate the coordinates of the vector $\xi$, using the formulas obtained for the summands on the right side of (17)

$$
\begin{aligned}
\xi= & \left(\left(-\varphi^{2}+\frac{1}{12} \varphi^{4}\right)+\frac{16}{3} \varphi^{4}+\left(2 \varphi^{2}-\frac{2}{3} \varphi^{4}\right)+O\left(\varphi^{6}\right),\right. \\
& \left.\left(2 \varphi-\frac{1}{3} \varphi^{3}\right)+\left(4 \varphi+\frac{8}{3} \varphi^{3}\right)+\left(2 \varphi-\frac{4}{3} \varphi^{3}\right)+O\left(\varphi^{5}\right)\right) \\
= & \left(\varphi^{2}+\frac{19}{4} \varphi^{4}+O\left(\varphi^{6}\right), 8 \varphi-\varphi^{3}+O\left(\varphi^{5}\right)\right) .
\end{aligned}
$$

We want to find the ratio of the lengths of segments $P S$ and $S L$, or equivalently, $\left|P_{32} S_{32}\right| /\left|S_{32} L_{32}\right|$, and to examine when this ratio is irrational. To do this, find the lengths of the vectors $\overrightarrow{B_{32} L_{32}}, \overrightarrow{K_{32} L_{32}}, \overrightarrow{M_{32} L_{32}}$, and $\overrightarrow{S_{32} L_{32}}$ in sequence, and then the lengths of the segments needed.

First calculate the coordinates of the vector $\overrightarrow{C_{0} L_{32}}$. Denote the second coordinate of vector a by $(\mathbf{a})$. Then

$$
\begin{aligned}
\overrightarrow{C_{0} L_{32}} & =\overrightarrow{C_{0} C_{43}} \cdot \frac{(\xi)_{y}-\frac{1}{2} \tan \frac{\varphi}{2}}{\left(\overrightarrow{C_{0} C_{43}}\right)_{y}} \\
& =\left(\frac{31}{4}-\frac{47}{48} \varphi^{2}+O\left(\varphi^{4}\right)\right)\left(\frac{21}{2}+\frac{13}{24} \varphi^{2}+O\left(\varphi^{4}\right)\right)^{-1} \cdot\left(\begin{array}{l}
\frac{3}{2} \varphi^{2}+\frac{89}{24} \varphi^{4}+O\left(\varphi^{6}\right) \\
\frac{21}{2} \varphi+\frac{13}{24} \varphi^{3}+O\left(\varphi^{5}\right)
\end{array}\right) \\
& =\left(\frac{31}{28} \varphi^{2}+\frac{17923}{7056} \varphi^{4}+O\left(\varphi^{6}\right), \frac{31}{4} \varphi-\frac{47}{48} \varphi^{3}+O\left(\varphi^{5}\right)\right)
\end{aligned}
$$

Now find the first of the vectors we need, $\overrightarrow{B_{32} L_{32}}$ :

$$
\begin{aligned}
\overrightarrow{B_{32} L_{32}} & =\overrightarrow{B_{32} B_{0}}+\overrightarrow{B_{0} C_{0}}+\overrightarrow{C_{0} L_{32}}=-\xi+\left(\begin{array}{c}
-\frac{1}{2} \\
\frac{1}{2} \tan \frac{\varphi}{2}
\end{array}\right)+\overrightarrow{C_{0} L_{32}} \\
& =\left(-\frac{1}{2}+\frac{3}{28} \varphi^{2}+\frac{41439}{7056} \varphi^{4}+O\left(\varphi^{6}\right), 0\right) .
\end{aligned}
$$

As expected, vector $\overrightarrow{B_{32} L_{32}}$ proved to be horizontal. To define vector $\overrightarrow{L_{32} M_{32}}$. draw a straight line through vertex $C_{30}$ parallel to vector $\overrightarrow{C_{0} L_{32}}$ and find the intersection of this line with the straight line $A_{32} B_{32}$. This will be the point $M_{32}$. 
Let $D$ be the intersection of the straight line $C_{0} C_{43}$ with the horizontal line passing through vertex $C_{30}$; then $\overrightarrow{D C_{30}}=\overrightarrow{L_{32} M_{32}}$. On the other hand, $\overrightarrow{D C_{30}}=\overrightarrow{C_{0} C_{30}}$ $-\overrightarrow{C_{0} D}$, so it remains to define vectors $\overrightarrow{C_{0} C_{30}}$ and $\overrightarrow{C_{0} D}$ in order to find vector $\overrightarrow{L_{32} M_{32}}$.

$$
\begin{aligned}
\overrightarrow{C_{0} C_{30}} & =\overrightarrow{C_{0} B_{0}}+\overrightarrow{B_{0} B_{32}}+\overrightarrow{B_{32} C_{30}}=\left(\begin{array}{c}
\frac{1}{2} \\
-\frac{1}{2} \tan \frac{\varphi}{2}
\end{array}\right)+\xi+\left(\begin{array}{c}
-\frac{1}{2} \\
-\frac{1}{2} \tan \frac{\varphi}{2}
\end{array}\right) \\
& =\left(\varphi^{2}+\frac{19}{4} \varphi^{4}+O\left(\varphi^{6}\right), \frac{15}{2} \varphi-\frac{23}{24} \varphi^{3}+O\left(\varphi^{5}\right)\right) .
\end{aligned}
$$

Vector $\overrightarrow{C_{0} D}$ is proportional to vector $\overrightarrow{C_{0} L_{32}}$ with coefficient given by

$$
\lambda=\frac{\left(\overrightarrow{C_{0} C_{30}}\right)_{y}}{\left(\overrightarrow{C_{0} L_{32}}\right)_{y}}=\left(\frac{15}{2} \varphi-\frac{23}{24} \varphi^{3}+O\left(\varphi^{5}\right)\right) \cdot\left(\frac{31}{4} \varphi-\frac{47}{48} \varphi^{3}+O\left(\varphi^{5}\right)\right)^{-1}
$$

i.e.,

$$
\begin{aligned}
\overrightarrow{C_{0} D} & =\lambda \cdot \overrightarrow{C_{0} C_{32}}=\lambda \cdot\left(\frac{31}{28} \varphi^{2}+\frac{17923}{7056} \varphi^{4}+O\left(\varphi^{6}\right), \frac{31}{4} \varphi-\frac{47}{48} \varphi^{3}+O\left(\varphi^{5}\right)\right) \\
& =\left(\frac{15}{14} \varphi^{2}+\frac{3611}{5208} \varphi^{4}+O\left(\varphi^{6}\right), \frac{15}{2} \varphi-\frac{23}{24} \varphi^{3}+O\left(\varphi^{5}\right)\right) .
\end{aligned}
$$

Fig. 9

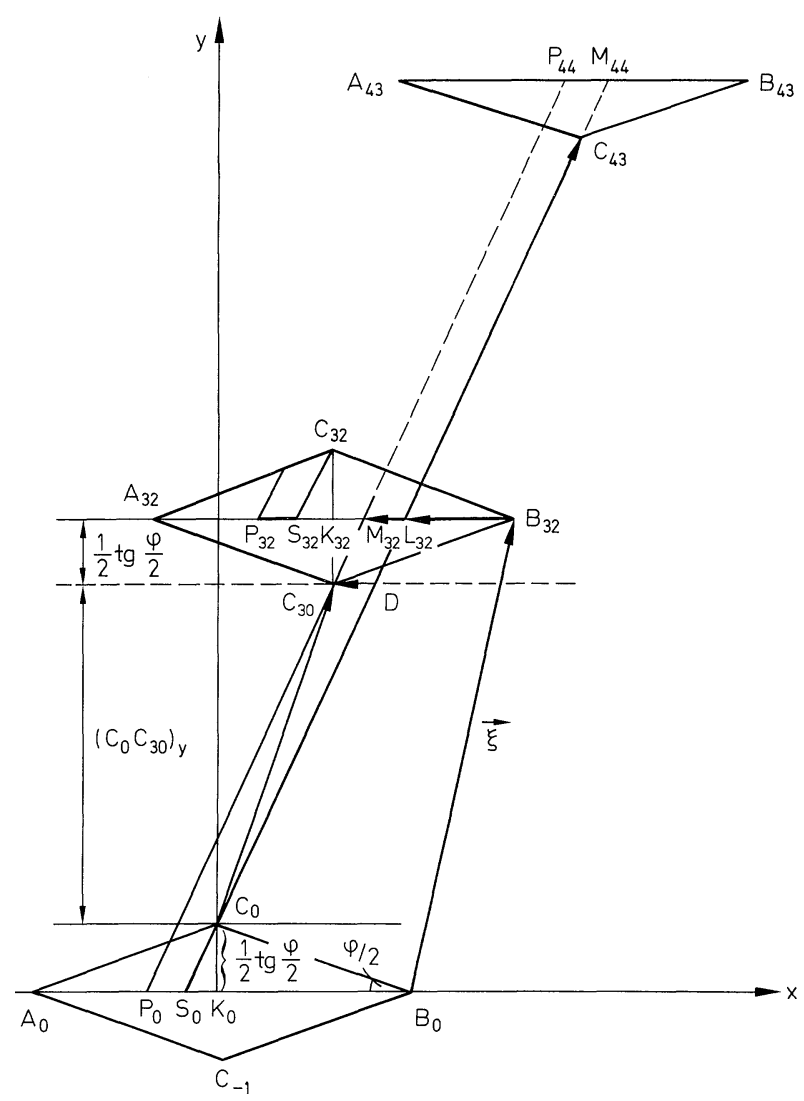


Finally, we obtain

$$
\overrightarrow{L_{32} M_{32}}=\overrightarrow{D C_{30}}=\overrightarrow{C_{0} C_{30}}-\overrightarrow{C_{0} D}=\left(-\frac{1}{14} \varphi^{2}+\frac{21127}{5208} \varphi^{4}+O\left(\varphi^{6}\right), 0\right) .
$$

Vector $\overrightarrow{L_{32} M_{32}}$ is also horizontal, as it should be.

Now we can calculate the lenths of the vectors we are interested in: $\overrightarrow{B_{32} L_{32}}$, $\overrightarrow{L_{32} M_{32}}, \overrightarrow{K_{32} M_{32}}$, and $\overrightarrow{S_{32} M_{32}}$. From formulas (20) and (23) and from Fig. 9, it follows that

$$
\begin{gathered}
\left|\overrightarrow{B_{32} L_{32}}\right|=\frac{1}{2}-\frac{3}{28} \varphi^{2}-\frac{41439}{7056} \varphi^{4}+O\left(\varphi^{6}\right), \\
\left|\overrightarrow{L_{32} M_{32}}\right|=\frac{1}{14} \varphi^{2}-\frac{21127}{5208} \varphi^{4}+O\left(\varphi^{6}\right), \\
\left|\overrightarrow{K_{32} M_{32}}\right|=\left|\overrightarrow{K_{32} L_{32}}\right|-\left|\overrightarrow{L_{32} M_{32}}\right|=\frac{1}{28} \varphi^{2}-\frac{1085501}{218736} \varphi^{4}+O\left(\varphi^{6}\right), \\
\left|\overrightarrow{S_{32} L_{32}}\right|=2\left|\overrightarrow{K_{32} M_{32}}\right|+\left|\overrightarrow{L_{32} M_{32}}\right|=\left(\frac{1}{14} \varphi^{2}-\frac{1085501}{109368} \varphi^{4}+O\left(\varphi^{6}\right)\right) \\
+\left(\frac{1}{14} \varphi^{2}-\frac{21127}{5208} \varphi^{4}+O\left(\varphi^{6}\right)=\frac{1}{7} \varphi^{2}-\frac{191146}{13671} \varphi^{4}+O\left(\varphi^{6}\right) .\right.
\end{gathered}
$$

Finally, calculate the ratio $\left|P_{32} S_{32}\right| /\left|S_{32} L_{32}\right|$ :

$$
\begin{aligned}
\left|P_{32} S_{32}\right| /\left|S_{32} L_{32}\right|= & \left(\frac{1}{14} \varphi^{2}-\frac{21127}{5208} \varphi^{4}+O\left(\varphi^{6}\right)\right)\left(\frac{1}{7} \varphi^{2}\right. \\
& \left.-\frac{191146}{13671} \varphi^{4}+O\left(\varphi^{6}\right)\right)^{-1}=\frac{1}{2}+\frac{321127}{109368} \varphi^{2}+O\left(\varphi^{4}\right) .
\end{aligned}
$$

\subsection{The End of Proof of Theorem 2}

It remains to prove that the ratio (25) is irrational for almost all $\varphi$ in some interval $\left(0, \varphi_{0}\right)$. Note that the function $h(\varphi)$ on the right side of $(25)$ is continuously differentiable and non-constant, from which the assertion of Theorem 2 follows.

\section{Proof of Theorem 3}

It follows from the proof of Theorem 2 that when the ratio of the lengths of segments $P S$ and $S L$ is irrational, any trajectory, the first link of which is parallel to segment $s$ and which lies between segments $\not$ and $s$, will fill a set everywhere densely in the isosceles triangle $A B C$; furthermore, in the straightened form this set fills two corridors, as shown in Fig. 8, these being the corridor between the parallel segments $S_{0} L_{32}$ and $P_{0} M_{32}$, and the corridor between the parallel segments $L_{32} M_{44}$ and $S_{32} P_{44}$. In every triangle $A_{i} B_{i} C_{i}$ these corridors will cut out one quadrilateral - a trapezoid, the lateral sides of which lie on the base and on a lateral side of the triangle $A_{i} B_{i} C_{i}$.

By returning triangles $A_{i} B_{i} C_{i}$ to the original triangle $A B C$ (i.e., by making reverse reflections from the corresponding sides of the triangles), we obtain region 65 (a non-convex polygon) in the original triangle $A B C$, which is the union of 44 such trapezoids (region $\mathbb{G}$ is roughly depicted in Fig. 10 ; it is not precise). Note that the number of sides of polygon $(\mathfrak{5}$ can be different for different values of the angle $\varphi$. 
Fig. 10

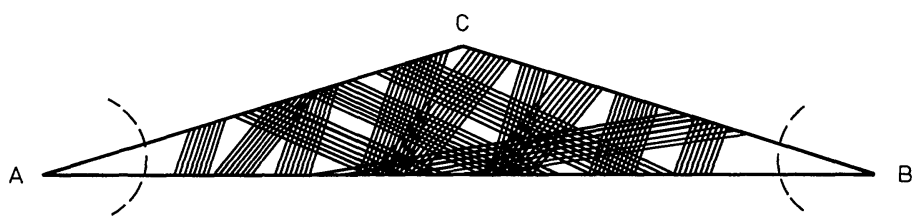

It is easy to see that there exists a number $\imath>0$, such that circular sectors of radius $\iota$ with centers at the points $A$ and $B$ ( $\imath$-neighborhoods of points $A$ and $B$ ) do not intersect the points of polygon $(\mathfrak{5}$. Thus there is a billiard trajectory, the first link of which is parallel to segment $s$ and belongs to polygon $\mathbf{6}$, which fills polygon $\mathbf{5}$ everywhere densely, and never falls into a $\iota$-neighborhood of vertices $A$ and $B$.

Finally, by making a last reflection of the left half of triangle $A B C$ in the segment $C K$, we obtain the right triangle we began with containing a polygonal region $\left(\mathfrak{G}^{\prime}\right.$ that is everywhere densely filled by a billiard trajectory, which avoids the $\imath$-neighborhood of the vertex with angle $\alpha . \mathfrak{b}^{\prime}$ is a union of trapezoids, the lateral sides of which lie on a cathetus and on the hypotenuse. These trapezoids cut out a finite number of non-intersecting segments $\kappa_{1}, \kappa_{2}, \ldots, \kappa_{N}$ on the hypotenuse.

Consider now the configuration space of the mechanical system of two point particles with masses $m$ and $\mathrm{km}$ on the interval $[0,1]$ - that is, the right triangle with angle $\alpha, \tan \alpha=k$ (without loss of generality, we assume $k<1$ ).

By virtue of Theorem 2, for almost all $\alpha$ in some interval $\left(0, \alpha_{0}\right)$, and hence for almost all $k$ in some interval $\left(0, k_{0}\right)$, the configuration trajectory fills polygon ${\left(\mathfrak{b}^{\prime}\right.}^{\prime}$ everywhere densely and, in particular, the configuration point fills the union of the segments $\kappa_{1}, \ldots, \kappa_{N}$ everywhere densely on the triangle's hypotenuse.

Any point on the segment $\kappa_{i}$ corresponds to a point where our particles collide with each other, which belongs to some segment $\delta_{i} \subset[0,1]$. Hence, the set of points where the particles collide is an everywhere dense set of points on $\delta_{1} \cup \delta_{2} \cup \ldots \cup \delta_{N}$; this is a union of a finite number of segments on $[0,1]$. Therefore Theorem 3 is proved.

\section{Proof of Theorem 4}

The polyhedron whose existence is asserted in Theorem 4 is a prism - the direct product of segment $\left[0, \frac{1}{2}\right]$, (the vertical generator of the prism) and the convex $n$-agon from Theorem 1 . When moving in the $n$-agon as described in the proofs of Theorems 1 and 2, a "flat" billiard trajectory fills some region $6 \mathfrak{5}$ in the $n$-agon everywhere densely. Here region $(\mathfrak{5}$ does not coincide with the whole $n$-agon. It is asserted that a vertical component $v$ along the prism's generator can be added to the velocity vector of this particle (the modulus of which is one), such that the new "three-dimensional" billiard trajectory will fill everywhere densely a threedimensional region in the prism, which does not coincide with the whole prism.

In order to prove this assertion, first trace the flat trajectory. Consider those trajectories from the bundle in Fig. 3 (or Fig. 7a), whose initial points belong to the segment $[0, a]$ of family $\mathfrak{U}$ 's cross-section $[0,1]$ (or to segment PS on triangle $A B C$ 's base $A B$ ). We know from the proof of Theorem 1 that segment $[0, a]$ turns 
into segment $[1-a, 1]$ under the action of the "billiard system." It is easy to see that the same time is required for every point of segment $[0, a]$ to reach segment $[1-a, 1]$. Denote this time by $T_{1}$. Analogously, all points of segment $[a, 1]$ reach segment $[0,1-a]$ in time $T_{2}$.

Now trace the trajectory of the three-dimensional particle in the prism. First, symmetrically reflect the prism from its upper base; we obtain a new prism, the generator of which is twice as large - it is $I=[0,1]$. In this prism, the particle moves with a vertical component of velocity $v$, and after reaching the upper base, it skips vertically downwards onto the lower base.

Consider the particle's intersections with the square

$$
\Pi \stackrel{\text { def }}{=}[0,1] \times I,
$$

which is orthogonal to the prism's base (the square lies in the prism's vertical cross-section). Then at time $T_{i}$ point $(x, y) \in \Pi$ will have travelled to the point

$$
\left(x+a ;\left(y+v T_{i}\right) \bmod 1\right) \in \Pi ; \quad i=1,2 .
$$

Here $a$ is the length of the segment $[0, a] \subset[0,1]$. Note that by choosing the billiard trajectory, $a$ can be irrational; rewrite $a=\Theta$.

Consider an arbitrary point $(x, y) \in \Pi$ and its subsequent images under the indicated transformation. If the set of these images fills the square $\Pi$ everywhere densely, then the billiard trajectory fills everywhere densely the three-dimensional region $\mathfrak{5} \times I$, which does not coincide with the whole prism. We must define the conditions under which $\Pi$ will be filled everywhere densely by images of the point $(x, y)$. In the following lemma we give the criterion for an everywhere dense filling of square $\Pi$ by these images.

Lemma. Let $\Theta$ be a positive, irrational number less than $1, T_{1}$ and $T_{2}$ be arbitrary positive numbers, and $x_{0}, y_{0} \in[0,1]$. Define the two sequences $\left(x_{n}\right)$ and $\left(y_{n}\right), n=0,1, \ldots$ by the following recurrent relations:

(a) $x_{n}=\left\{x_{0}+\Theta n\right\}$, where $\{\cdot\}$ is the fractional part of a number,

(b) $y_{n}=\left\{\begin{array}{lll}\left(y_{n-1}+v T_{1}\right) \bmod 1 & \text { if } & x_{n-1}+\Theta \leqq 1 \\ \left(y_{n-1}+v T_{2}\right) \bmod 1 & \text { if } & x_{n-1}+\Theta>1\end{array}\right.$

We assert that the set of points $\left(x_{n}, y_{n}\right) \in[0,1] \times[0,1]=\Pi$ fills $\Pi$ everywhere densely if, and only if

i) the number $\gamma=v\left(T_{2}+\left(T_{1}-T_{2}\right) \Theta\right)$ is irrational, and

ii) the numbers $1, \Theta, \gamma$ are rationally independent (i.e., $n_{1} \cdot 1+n_{2} \cdot \Theta+n_{3} \gamma \neq 0$ with any integers $\left.n_{1}, n_{2}, n_{3}\right)$.

Proof of Lemma. Define $\alpha_{1}=\left(v T_{1}\right) \bmod 1, \alpha_{2}=\left(v T_{2}\right) \bmod 1$. Then on the twodimensional torus Tor $^{2}=S^{1} \times S^{1}$, obtained by identifying the opposite sides of square $\Pi$, relations (a) and (b) give the following transformation $P$ :

$$
P:(x, y) \rightarrow\left(x+\Theta, y+\alpha_{2}+\left(\alpha_{1}-\alpha_{2}\right) \chi_{[0, \Theta]}(x)\right),
$$

where $\chi_{[0, \Theta]}(x)$ is the indicator of segment $[0, \Theta]$. Define

$$
g(x)=\alpha_{2}+\left(\alpha_{1}-\alpha_{2}\right) \chi_{[0, \Theta]}(x) \text {. }
$$


Then

$$
P(x, y)=(x+\Theta, y+g(x)),
$$

i.e., $P$ is a skew shift on torus $\operatorname{Tor}^{2}$ (see [5]). Let $C$ be another skew shift on this torus:

$$
C(x, y)=(x, y+\varphi(x))
$$

where $\varphi(x)$ is some function on the circle $S^{1}$.

Consider the transformation $R=C P C^{-1}$. Write it explicitly:

$$
\begin{gathered}
C^{-1}(x, y)=(x, y-\varphi(x)) \\
P C^{-1}(x, y)=(x+\Theta, y-\varphi(x)+g(x)), \\
C P C^{-1}(x, y)=(x+\Theta,(y-\varphi(x)+g(x))+\varphi(x+\Theta)) .
\end{gathered}
$$

Thus,

$$
R(x, y)=(x+\Theta, y+g(x)+\varphi(x+\Theta)-\varphi(x)) .
$$

Define $\gamma=\alpha_{2}+\left(\alpha_{1}-\alpha_{2}\right) \Theta$. Assume that the transformation $C$ is such that

$$
R(x, y)=(x+\Theta, y+\gamma),
$$

i.e., $R$ is a shift on the torus Tor ${ }^{2}$. Then it follows from the equalities $R^{k}=C P^{k} C^{-1}$, $R^{k} C=C P^{k}$ that for any point $z=(x, y)$,

$$
C\left(P^{k} z\right)=R^{k}(C z)
$$

and the trajectory $\left\{P^{k} z\right\}_{k=0}^{\infty}$ of the point $z$ coincides with the trajectory $\left\{R^{k}(C z)\right\}_{k=0}^{\infty}$ "shifted" with the help of $C^{-1}$ :

$$
\left\{P^{k} z\right\}_{k=0}^{\infty}=C^{-1}\left\{R^{k}(C z)\right\}_{k=0}^{\infty} .
$$

From this it follows that the trajectory $\left\{P^{k} z\right\}_{k=0}^{\infty}$ is everywhere dense on the torus if, and only if, the trajectory $\left\{R^{k} z\right\}_{k=0}^{\infty}$ is everywhere dense on the torus, and the latter holds by virtue of (29) when lemma conditions (i) and (ii) hold.

Thus, it remains to reduce expression (28) to the form of (29); in other words, we must solve the following equation:

$$
g(x)+\varphi(x+\Theta)-\varphi(x)=\gamma .
$$

By substituting $g(x)$ in the left side, we obtain

$$
\begin{gathered}
\alpha_{2}+\left(\alpha_{1}-\alpha_{2}\right) \chi_{[0, \Theta]}(x)+\varphi(x+\Theta)-\varphi(x)=\alpha_{2}+\left(\alpha_{1}-\alpha_{2}\right) \Theta \\
\chi_{[0, \Theta]}(x)=\underset{\Downarrow}{\Theta}+\left(\alpha_{2}-\alpha_{1}\right)^{-1}(\varphi(x+\Theta)-\varphi(x)) \\
\chi_{[0, \Theta]}(x)=\Theta+f(x+\Theta)-f(x) .
\end{gathered}
$$

We define $f(x)=\left(\alpha_{2}-\alpha_{1}\right)^{-1} \varphi(x)$. In the theory of skew shifts, Eq. (30) is a homologous equation.

It is easy to see that the function

$$
f(x)=-\{x-\Theta\}
$$


where $\{\cdot\}$ is the fractional part of a number, is the solution of Eq. (30). It can be obtained by expanding both sides of Eq. (30) into a Fourier series and comparing the corresponding Fourier coefficients.

This proves the lemma and also Theorem 4.

\section{Proof of Theorem 5}

Since the trajectory $\Gamma$ fills region $\Omega$ everywhere densely, it is sufficient to prove that in an arbitrary $\varepsilon$-neighborhood of a point $q \in \Gamma$, the set of periodic points is everywhere dense, and the periodic trajectories passing through these points are at an angle $<\varepsilon$ to $\Gamma$.

Trajectory $\Gamma$ is a special one (see the construction in the proofs of Theorems 1 and 2). Its first link belongs to that family $\mathfrak{U}$ of parallel segments, whose crosssection $[0,1]$ undergoes an exchange transformation $f$ of the two segments $[0, a]$ and $[a, 1]$, i.e., $\forall x \in[0,1] f(x)=(x+b) \bmod 1$, where $b=1-a$, or in another form, $f(x)=(x-a) \bmod 1$, where the number $a$ is irrational. We will denote the $r^{\text {th }}$ exchange transformation image of some object $x$ (a point, a segment, etc.) by $f^{i}(x)$ if $r \geqq 0$, and the $r^{\text {th }}$ preimage of $x$ if $r<0$, also by $f^{\imath}(x)$.

Consider the preimages $f^{-k}(a)$ of the point $a$ on segment $[0,1], k=0,1, \ldots$ It is obvious that $f^{-k}(a)=\{(k+1) a\}$, where $\{\cdot\}$ is the fractional part of a number. All these preimages are different for different $k$ and they fill the segment $[0,1]$ everywhere densely when $k \rightarrow \infty$.

If $k$ runs through only a finite set of values $0,1,2, \ldots, n-1$, the numbers $\{(k+1) a\}$ divide $[0,1]$ into $n+1$ non-intersecting (except for their endpoints) segments which we will designate as $\left[0, \alpha_{1}\right],\left[\alpha_{1}, \alpha_{2}\right], \ldots,\left[\alpha_{n-1}, \alpha_{n}\right],\left[\alpha_{n}, 1\right]$. Consider only those values of $n$ for which the the leftmost segment is $[0,\{n a\}]$, and such that $\{n a\}$ is less than the length of any of the remaining $n$ segments (we consider an $n$ for which point $a$, bouncing rightwards, jumps over point 1 and turns out to be near the left endpoint 0 of segment $[0,1]$ ). We prove that the set of such $n$ 's is infinite.

Indeed, decompose the number $a$ into a continued fraction and let $p_{\diamond} / q_{\diamond}$, $\checkmark=1,2, \ldots$, be convergents of the continued fraction $\left(p_{s} / q_{s} \rightarrow a\right.$ when $\left.s \rightarrow \infty\right)$. It is known from the theory of continued fractions (see [6]) that convergents are the best approximations to the number $a ; a-p_{s} / q_{s}<0$ when $s$ is odd and $a-p_{s} / q_{s}>0$ when $s$ is even. From this it follows that the numbers $\left\{a q_{s}\right\}=\left\{a q_{s}-p_{s}\right\}$ and $\left\{a q_{s+1}\right\}$ $=\left\{a q_{s+1}-p_{\lrcorner+1}\right\}$, which are interpreted as points on the circle $S^{1}$, obtained by pasting together the endpoints 0 and 1 of segment $[0,1]$, lie on different sides of the point 0 and that one of the arcs $\left(0,\left\{a q_{s}\right\}\right)$ and $\left(0,\left\{a q_{s+1}\right\}\right)$ has minimal length among the remaining arcs into which the circumference is divided. If, for instance, point $\left\{a q_{s+1}\right\}$ is closer to 0 than point $\left\{a q_{s}\right\}$, replacing o by $\delta+1$, we obtain (from the theory of continued fractions) that point $\left\{a q_{s+2}\right\}$ is closer to 0 than point $\left\{a q_{s+1}\right\}$ (and the distance from $\left\{a q_{s+2}\right\}$ to 0 will be less than the lengths of all newly created arcs on $S^{1}$ ). Therefore, by taking the denominators of the convergents of the number $a$ as $n$, we will obtain the required assertion for an infinite subset of this set. 
Choose any $n$ from the set indicated. Choose an arbitrary point $\beta$ on an arbitrary segment of the partition $\left[\alpha_{i}, \alpha_{i+1}\right]$, such that $\beta$ is at least the distance $\{n a\}$ from the segment's right endpoint, and consider the segment

$$
[\beta, \beta+\{n a\}] \subset\left[\alpha_{i}, \alpha_{i+1}\right] .
$$

Denote this segment by $\Delta_{i}(\beta)$.

Let us see what happens to $\Delta_{i}(\beta)$ under the iterations of the exchange transformation $f$. It is easy to see that by our choice of $n$, segment $f^{k}\left(\Delta_{i}(\beta)\right)$ is of length $\{n a\}$ for all $k, 1 \leqq k \leqq n$, and lies fully within one of the segments $\left[\alpha_{j}, \alpha_{j+1}\right]$ of the partition. Since the endpoints of the partition's segments are preimages of the point $a$, we find that none of the preimages of point $a$, including the $n^{\text {th }}$ one, belong to the segment $f^{k}\left(\Delta_{i}(\beta)\right)$. The last segment

$$
f^{n}\left(\Delta_{i}(\beta)\right)=\left[f^{n}(\beta) ; f^{n}(\beta+\{n a\})\right]=\left[f^{n}(\beta) ; \beta\right]
$$

adjoins the segment $\Delta_{i}(\beta)$ at its right endpoint.

Now consider all the trajectories of family $\mathfrak{A}$ leaving segment $\Delta_{i}(\beta)$ and straighten them out in accordance with the procedure for straightening billiard trajectories. As a result, we obtain a bundle of parallel trajectories reflecting from the same sides of polygon $Q$, including the $n^{\text {th }}$ iteration of segment $\Delta_{i}(\beta)$. Indeed, by virtue of the above reasoning, this bundle cannot pass through any polygon vertex in the number of reflections mentioned.

Figure 11 shows how the straightened bundle, which intersects the sides of polygon $Q$ from which it reflects, transfers segment $\Delta_{i}(\beta)$ on one cross-section of family $\mathfrak{A}$ into segment $f^{n}\left(\Delta_{i}(\beta)\right)$, which lies on another cross-section of family $\mathfrak{A}$. A parallelogram with bases $\Delta_{i}(\beta)$ and $f^{n}\left(\Delta_{i}(\beta)\right)$ forms as a result of the straightening; the lateral sides of the parallelogram are parallel to the straightened trajectory $\Gamma$. It is easy to see that the diagonal of this parallelogram which connects points $\beta$ and $f^{n}(\beta+\{n a\})$,

(1) is a straightened periodic trajectory in $Q$, and

(2) reflects from the same sides of $Q$ as the bundle.

It is clear that when $n \rightarrow \infty$ the set of points $\beta$ will fill segment $[0,1]$ everywhere densely, the lengths of the segments will tend to zero, and the angle between the base of the parallelogram considered and its diagonal will also tend to zero (since the angle between the straightened trajectory and the cross-section of family $\mathfrak{A}$ is

Fig. 11

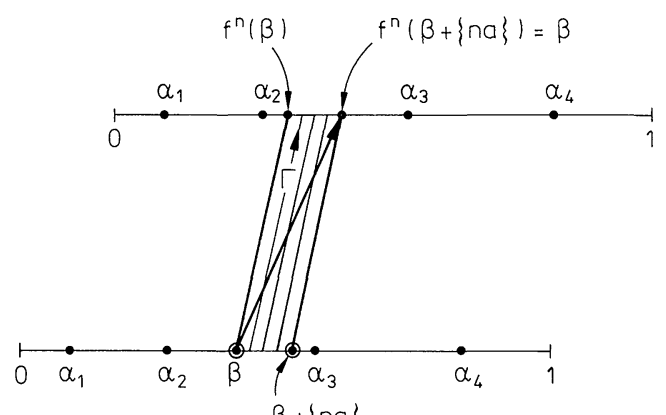

$\beta+\{n a\}$ 
fixed and when $n \rightarrow \infty$ the parallelogram stretches along $\Gamma$ and shrinks along the cross-section of $\mathfrak{U}$ ).

Hence, the periodic trajectory can differ by as little as desired from the trajectory $\Gamma$ and thus region $\Omega$ contains an everywhere dense set of periodic points $q \in Q$.

\section{Remarks and Hypotheses}

1. From the proofs of Theorems 1 and 2 we can see that in some convex polygon a non-periodic and not everywhere dense billiard trajectory can be constructed according to any aperiodic exchange transformation of two segments. Can an analogous billiard trajectory be constructed in some convex polygon according to some given aperiodic exchange transformation of three or more segments?

It turns out that we can construct a convex polygon with such a trajectory according to any aperiodic exchange transformation of $n \geqq 3$ segments. We will not submit the proof here, due to the awkwardness of the exact construction; we shall instead argue plausibly using mirrors and rays of light. These arguments can be made conclusive.

Place horizontally the segment $[0,1]$, divided into the $n$ segments. Emit upwards a beam of parallel light rays and regard it as a union of $n$ beams from each segment. After a complex motion of these $n$ beams in the polygon, which we will describe, they return parallel to each other onto the segment $[0,1]$ from below, transformed in accordance with the given permutation. This can be visualized in the following way:

First, $n$ rising beams (i.e., the whole beam) reflect from a hyperbolic concave mirror of small curvature and then separate (the mirror will later be replaced by neighboring flat mirrors). At a sufficiently large distance from the separation point we can place $n$ flat mirrors which effect the given permutation $\pi$; after that, with the help of another $n$ mirror, transfer the beams onto a hyperbolic concave mirror placed below segment $[0,1]$ (also to be replaced by flat mirrors). The lower mirror unites all $n$ beams into one parallel beam which lands on segment $[0,1]$ from below. Then, the required polygon is completed with the mirrors. All these mirrors can be set in such a way that the polygon obtained will be convex.

If the polygon appears to be rational (which can be achieved by small shifts), then the billiard trajectories, when dislocated on the manifold constructed according to the polygon (on a sphere with a large number of handles), will everywhere densely fill a submanifold $\mathscr{R}$ with an edge, as described below.

Consider a rectangle, the upper base of which is divided into $n$ oriented segments $1,2, \ldots, n$ and the lower base divided into $n$ segments similarly oriented: $\pi(1), \pi(2), \ldots, \pi(n)$. From every segment $i$ emit a rectangular strip ("ribbon") obtained by the movement of segment $i$ till it reaches segment $\pi(i)$ (see the remark at the end of Sect. 2). Doing this, we obtain a two-dimensional oriented surface with a certain number of holes cut out of it. The billiard trajectory fills this surface everywhere densely. It flows past the holes as shown in Fig. 6, right. The remaining part of the manifold is free of this trajectory. 
2. It seems that in an $n$-dimensional simplex $\left\{\left(x_{1}, \ldots, x_{n}\right): 0 \leqq x_{i} \leqq \sqrt{m_{i}}, \frac{x_{i}}{\sqrt{m_{i}}}\right.$ $\left.\leqq \frac{x_{i+1}}{\sqrt{m_{i+1}}}\right\}$ which is the configuration space of a dynamical system of $n$ particles with masses $m_{1}, m_{2}, \ldots, m_{n}$ on the segment $[0,1]$, with elastic reflections, as in a right triangle, there exists a non-periodic and not everywhere dense billiard trajectory. (I do not known the proof.)

3. If the previous assertion is true, then for some initial conditions, the points of the particle collisions fill everywhere densely the union of a finite number of non-intersecting segments on $[0,1]$, apparently as in the case of two particles. Generally speaking, this assertion is not an automatic corollary from the assertion of the previous section, since the fact that a trajectory is everywhere dense in some region $(\mathfrak{5}$ does not mean that the points of this trajectory's reflection from the boundary $\partial \mathfrak{6} \mathfrak{5}$ fill $\partial \mathfrak{G}$ everywhere densely. (See the hexagon example in the proof of Theorem 1.)

4. We can pose the following questions in connection with the construction described in the proof of the lemma in Sect. 5. Consider the following dynamical system on the torus Tor $^{2}=S^{1} \times S^{1}$ pasted from the square $[0,1] \times[0,1]: n$ vertical flows advance along the vertical layer of $[0,1]$ with different velocities $v_{1}, \ldots, v_{n}$; and the base $S^{1}$ revolves by an irrational angle $\Theta$ (in the lemma of Sect. 5 there were two flows; the dividing line was $x=\Theta$ ). How does an arbitrary point behave under this transformation? What is the criterion for the images of this point to be everywhere dense on Tor $^{2}$ ?

5. Note that the prism constructed in the proof of Theorem 4 can be considered as the configuration space of the following dynamical system:

Consider two 2-dimensional elastic balls (disks) of small diameter $D$ with masses $m_{1}$ and $m_{2}$, located at the same vertical height at an initial time in the square $[0,1] \times[0,1]$ with elastic walls. Let the initial velocities of these balls be $\mathbf{V}_{1}$ and $\mathbf{V}_{2},\left(\mathbf{V}_{1}\right)_{y}=\left(\mathbf{V}_{2}\right)_{y}=v,\left(\mathbf{V}_{1}\right)_{x}=v_{1},\left(\mathbf{V}_{2}\right)_{x}=v_{2}$ (Fig. 12a).

It is easy to see that at each moment of time the balls will be at the same height; the vertical components of their velocities will be the same and will equal either $v$ or $(-v)$; the horizontal components will be redistributed according to the laws of an elastic collision when the balls collide; the horizontal components will change signs when the balls reflect from the side walls. Thus, our dynamical system is a direct product of two independent systems and its configuration space is a direct product of the corresponding configuration spaces : a right triangle and a segment, i.e., a three-dimensional prism. [Generally speaking, the configuration space of

Fig. 12a and b

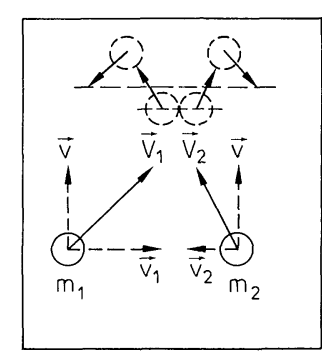

a)

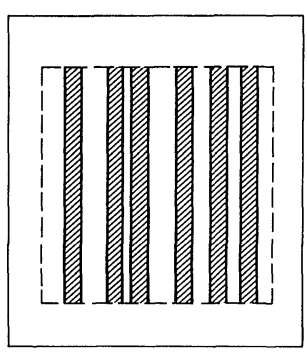

b) 
two balls in a square is a four-dimensional manifold, the intersection of the interior of a four-dimensional cube and the complement of the cylinder $\left(x_{1}-x_{2}\right)^{2}$ $+\left(y_{1}-y_{2}\right)^{2} \leqq D^{2}$ in $\mathbb{R}^{4}$ (see [7, Part II] for details). However, in the given case the configuration point moves only in the three-dimensional cross-section of the fourdimensional manifold - the prism indicated.]

From the results of Theorems 3 and 4 we promptly obtain the following

Assertion. For almost all $k=\frac{m_{1}}{m_{2}}$ in some interval $\left(0, k_{0}\right)$ in the described mechanical system of elastic balls in a square, and for some initial state of the balls (the positions and velocities $v, v_{1}, v_{2}$ ), the set of points where the balls collide is everywhere dense in the union of a finite number of non-intersecting rectangles (Fig. 12b).

6. It seems that Theorem 5 of Sect. 6 can be made stronger. Call a point $(q, v)$ of phase space $\mathscr{M}=Q \times S^{1}$ periodic if a periodic trajectory passes through it in $\mathscr{M}$.

Hypothesis 1. The set of periodic points is everywhere dense in some region $\Omega$ of $\mathscr{M}$.

It is natural to generalize Hypothesis 1 to the following:

Hypothesis 2. For any polygon $Q$ the set of periodic trajectories is everywhere dense in the whole phase space $\mathscr{M}$.

7. Do there exist polygons in which any billiard trajectory is either periodic or everywhere dense (i.e., no trajectories considered in this paper)? It seems to me the following hypotheses deal with that question.

Hypothesis 3 . For any $n \geqq 3$ there exists a convex $n$-agon in which any non-periodic trajectory of a particle is everywhere dense.

This hypothesis can be reinforced.

Hypothesis 4. For any $\varepsilon>0$ and any convex $n$-agon $Q_{n}$ there exists a convex $n$-agon $Q_{n}^{*}$, the angles of which differ from the corresponding angles of polygon $Q_{n}$ by less than $\varepsilon$, and in which all non-periodic trajectories are everywhere dense.

\section{References}

1. Zemlyakov, A.N., Katok, A.B.: Topological transitivity of billiards in polygons. Mat. Zametki 18, 291-300 (1975)

2. Boldrighini, C., Keane, M., Marchetti, F.: Billiards in polygons. Ann. Prob. 6, 532-540 (1978)

3. Zemlyakov, A.N.: Billiards and surfaces. Kvant 9, 2-9 (1979)

4. Sinai, Ya.G.: An introduction to ergodic theory. Moscow: Erivan (Lecture 10), 1976

5. Kornfeld, I.P., Sinai, Ya.G., Fomin, S.V.: Ergodic theory. Moscow: Nauka 1980

6. Khinchin, A.Ya.: Continued fractions. Moscow 1961

7. Galperin, G.A.: On systems of locally interacting and repelling particles moving in space. Trudy MMO, 43, 142-196 (1981)

Communicated by Ya. G. Sinai

Received March 15, 1983 
